\title{
Geometry of Hamiltonian chaos
}

Horwitz, Lawrence; Zion, Yossi Ben; Lewkowicz, Meir; Schiffer, Marcelo; Levitan, Jacob

Published in:

Physical Review Letters

Link to article, DOI:

10.1103/PhysRevLett.98.234301

Publication date:

2007

Document Version

Publisher's PDF, also known as Version of record

Link back to DTU Orbit

Citation (APA):

Horwitz, L., Zion, Y. B., Lewkowicz, M., Schiffer, M., \& Levitan, J. (2007). Geometry of Hamiltonian chaos. Physical Review Letters, 98(23), 234301. https://doi.org/10.1103/PhysRevLett.98.234301

\section{General rights}

Copyright and moral rights for the publications made accessible in the public portal are retained by the authors and/or other copyright owners and it is a condition of accessing publications that users recognise and abide by the legal requirements associated with these rights.

- Users may download and print one copy of any publication from the public portal for the purpose of private study or research.

- You may not further distribute the material or use it for any profit-making activity or commercial gain

- You may freely distribute the URL identifying the publication in the public portal

If you believe that this document breaches copyright please contact us providing details, and we will remove access to the work immediately and investigate your claim 


\title{
Geometry of Hamiltonian Chaos
}

\author{
Lawrence Horwitz, ${ }^{1,2,3}$ Yossi Ben Zion, ${ }^{1,3}$ Meir Lewkowicz, ${ }^{1}$ Marcelo Schiffer, ${ }^{1}$ and Jacob Levitan ${ }^{1,4}$ \\ ${ }^{1}$ Department of Physics, College of Judea and Samaria, Ariel 44837, Israel \\ ${ }^{2}$ School of Physics, Tel Aviv University, Ramat Aviv 69978, Israel \\ ${ }^{3}$ Department of Physics, Bar Ilan University, Ramat Gan 52900, Israel \\ ${ }^{4}$ Department of Physics, Technical University of Denmark, Lyngby 2800, Denmark
}

(Received 23 January 2007; published 4 June 2007)

\begin{abstract}
The characterization of chaotic Hamiltonian systems in terms of the curvature associated with a Riemannian metric tensor in the structure of the Hamiltonian is extended to a wide class of potential models of standard form through definition of a conformal metric. The geodesic equations reproduce the Hamilton equations of the original potential model when a transition is made to an associated manifold. We find, in this way, a direct geometrical description of the time development of a Hamiltonian potential model. The second covariant derivative of the geodesic deviation in this associated manifold results in (energy dependent) criteria for unstable behavior different from the usual Lyapunov criteria. We discuss some examples of unstable Hamiltonian systems in two dimensions.
\end{abstract}

DOI: 10.1103/PhysRevLett.98.234301

PACS numbers: 45.20.Jj, 05.45.Gg

A Hamiltonian system of the form (we use the summation convention)

$$
H=\frac{1}{2 M} g_{i j} p^{i} p^{j},
$$

where $g_{i j}$ is a function of the coordinates alone, has unstable orbits if the curvature associated with the metric $g_{i j}$ is negative. One can easily see that the orbits described by the Hamilton equations for (1) coincide with the geodesics on a Riemannian space associated with the metric $g_{i j}$, $[1,2]$; i.e., it follows directly from the Hamilton equations associated with (1) that (using (12) and the time derivative of (10))

$$
\ddot{x}_{\ell}=-\Gamma_{\ell}^{m n} \dot{x}_{m} \dot{x}_{n},
$$

where the connection form $\Gamma_{\ell}^{m n}$ is given by

$$
\Gamma_{\ell}^{m n}=\frac{1}{2} g_{\ell k}\left\{\frac{\partial g^{k m}}{\partial x_{n}}+\frac{\partial g^{k n}}{\partial x_{m}}-\frac{\partial g^{n m}}{\partial x_{k}}\right\},
$$

and $g^{i j}$ is the inverse of $g_{i j}$.

The second covariant derivative of the geodesic deviation depends on the curvature $[2,3]$

$$
R_{i}^{j k \ell}=\frac{\partial \Gamma_{i}^{j k}}{\partial x_{\ell}}-\frac{\partial \Gamma_{i}^{j \ell}}{\partial x_{k}}+\Gamma_{m}^{j k} \Gamma_{i}^{\ell m}-\Gamma_{m}^{j \ell} \Gamma_{i}^{k m}
$$

i.e., for $\xi_{i}=x_{i}^{\prime}-x_{i}$ on closely neighboring trajectories at $t$

$$
\frac{D^{2} \xi_{i}}{D t^{2}}=R_{i}^{j \ell k} \dot{x}_{j} \dot{x}_{k} \xi_{\ell}
$$

where $D / D t$ is the covariant derivative along the line $x_{j}(t)$. The sign of the scalar contraction of (4) then gives information on the stability of the orbits [3].

In this Letter, we point out that this formulation of dynamic stability has application to a much wider range of Hamiltonian models; in fact, every potential model Hamiltonian of the form

$$
H=\frac{p^{i 2}}{2 M}+V(x)
$$

where $V$ is a function of space variables alone, can be put into the form (1), where the metric tensor is of conformal form [4]. We obtain in this way a direct geometrical description of the time development for a Hamiltonian potential model.

Casetti, Pettini, and collaborators [5], for example, have studied the application of both the Jacobi and Eisenhardt metrics in their analyses of the geometry of Hamiltonian chaos. The Jacobi metric [1] (of the form $(E-V) \delta_{i j}$ ) leads to geodesic equations parametrized by the invariant distance associated with this metric on the manifold, in this case, the kinetic energy, thus corresponding to the Hamilton action. Transformation to parametrization by the time $t$ leads to the second order Newton law [5] in the form (14) below, for which the geometrical structure is no longer evident.

The Eisenhardt metric, leading to geodesic motion in $t$, involves the addition of an extra dimension. As noted by Caini et al. [5], this metric leads to the tangent dynamics commonly used to measure Lyapunov exponents in standard Hamiltonian systems. The method that we use, associated with a curvature that is explicitly energy dependent, appears to be a more sensitive diagnostic than the computation of exponents of a locally linearized system.

The formulation of Hamiltonian dynamics of the type of Eq. (6) in the form (1) is carried out by requiring that (6) be equivalent to (1). For a metric of conformal form

$$
g_{i j}=\varphi \delta_{i j}
$$

on the hypersurface defined by $H=E=$ const, the requirement of equivalence implies that 


$$
\varphi=\frac{E}{E-V(x)} .
$$

Substituting this result in the geodesic Eqs. (2), one obtains an equation that does not coincide in form with the Hamilton equations obtained from (6).

To see that the Hamilton equations obtained from (1) can, however, be put into correspondence with those obtained from the Hamiltonian of the potential model (6), we first note, from the Hamilton equations for (1), that

$$
\dot{x}_{i}=\frac{\partial H}{\partial p^{i}}=\frac{1}{M} g_{i j} p^{j} .
$$

We then use the geometrical property that $\dot{x}_{i}$ is a first rank tensor (as is $p^{i}$ ), under local diffeomorphisms that preserve the constraint that $H$ be constant, to define the velocity field

$$
\dot{x}^{j} \equiv g^{j i} \dot{x}_{i}=\frac{1}{M} p^{j},
$$

coinciding formally with one of the Hamilton equations implied by (6). From this definition, we recognize that we are dealing with two manifolds, each characterized, as we shall see, by a different connection form, but related by

$$
d x^{j}=g^{j i} d x_{i}
$$

on a common tangent space at each point (for which $g^{i j}$ is nonsingular).

To complete our correspondence with the dynamics induced by (6), consider the Hamilton equation for $\dot{p}^{i}$,

$$
\dot{p}^{\ell}=-\frac{\partial H}{\partial x_{\ell}}=-\frac{1}{2 M} \frac{\partial g_{i j}}{\partial x_{\ell}} p^{i} p^{j} .
$$

With the form (7) for $g^{i j}$, we obtain in the particular coordinate system in which (6) is defined,

$$
\dot{p}^{\ell}=-\frac{E}{E-V} \frac{\partial V}{\partial x_{\ell}} .
$$

Considering (11) as a change of variables, (13) becomes

$$
\dot{p}^{\ell}=-\frac{\partial V}{\partial x^{\ell}},
$$

the second Hamilton equation in the usual form, where $V$ is considered a function of the $\left\{x^{\ell}\right\}$, now considered as independent variables.

As a coordinate space, the $\left\{x^{\ell}\right\}$, which we shall call the Hamilton manifold, is not uniquely defined in terms of the original manifold $\left\{x_{\ell}\right\}$, which we shall call the Gutzwiller manifold, since (11) is not an exact differential. As we have remarked, we shall be working with two manifolds (characterized by the connection forms (3) and (21)). It is the local relation (11) which induces, from the geometry of the Gutzwiller manifold, a corresponding geometry on the Hamilton manifold. We shall discuss applications and interpretation of the physics of the Gutzwiller manifold elsewhere, but turn now to a further examination of the consequences of the relations (10) and (11).

The geodesic Eq. (2) can be transformed directly from an equation for $\ddot{x}_{j}$ to an equation for $\ddot{x}^{j}$, the motion defined in the Hamilton manifold. From (10), it follows that

$$
\begin{aligned}
\ddot{x}_{\ell} & =g_{\ell j} \ddot{x}^{j}+\frac{\partial g_{\ell j}}{\partial x_{n}} \dot{x}_{n} \dot{x}^{j} \\
& =-\frac{1}{2} g_{\ell k}\left\{\frac{\partial g^{k m}}{\partial x_{n}}+\frac{\partial g^{k n}}{\partial x_{m}}-\frac{\partial g^{n m}}{\partial x_{k}}\right\} \dot{x}_{m} \dot{x}_{n} .
\end{aligned}
$$

Now, using the identity

$$
\frac{\partial g_{\ell j}}{\partial x_{n}}=-g_{\ell k} \frac{\partial g^{k m}}{\partial x_{n}} g_{m j},
$$

it follows that, with the symmetry of $\dot{x}_{n} \dot{x}_{m}$,

$$
\frac{\partial g_{\ell j}}{\partial x_{n}} \dot{x}_{n} \dot{x}^{j}=-\frac{1}{2} g_{\ell k}\left(\frac{\partial g^{k m}}{\partial x_{n}}+\frac{\partial g^{k n}}{\partial x_{m}}\right) \dot{x}_{n} \dot{x}_{m} .
$$

Thus, the term on the left side of (15) containing the derivative of $g_{\ell j}$ cancels the first two terms of the connection form; multiplying the result by the inverse of $g_{\ell j}$, and applying the identity (16) to lower the indices of $g^{n m}$ in the remaining term on the right side of (15), one obtains

$$
\ddot{x}^{\ell}=-M_{m n}^{\ell} \dot{x}^{m} \dot{x}^{n},
$$

where

$$
M_{m n}^{\ell} \equiv \frac{1}{2} g^{\ell k} \frac{\partial g_{n m}}{\partial x^{k}} .
$$

Equation (18) has the form of a geodesic equation, with a truncated connection form. In fact, it can be shown (a full proof will be given elsewhere) that the form (19) is indeed a connection form, transforming as

$$
M_{m n}^{\prime \ell}=\frac{\partial x^{\prime \ell}}{\partial x^{r}} \frac{\partial x^{p}}{\partial x^{\prime m}} \frac{\partial x^{q}}{\partial x^{\prime n}} M_{p q}^{r}+\frac{\partial x^{\prime \ell}}{\partial x^{r}} \frac{\partial^{2} x^{r}}{\partial x^{\prime m} \partial x^{\prime n}},
$$

consistent with the covariance of (18) under local diffeomorphisms of the Hamilton manifold.

Substituting (7) and (8) into (18) and (19), the Kronecker deltas identify the indices of $\dot{x}^{m}$ and $\dot{x}^{n}$; the resulting square of the velocity cancels a factor of $(E-V)^{-1}$, leaving the Hamilton-Newton law (14). Equation (18) is therefore a covariant form of the Hamilton-Newton law, exhibiting what can be considered an underlying geometry of standard Hamiltonian motion.

The geometrical structure of the Hamilton manifold can be understood as follows. Let us write the covariant derivative for a (rank one) covariant tensor on the Gutzwiller manifold (defined as transforming in the same way as $\left.\partial / \partial x_{m}\right)$, using the full connection form (3),

$$
A^{m ; q}=\frac{\partial A^{m}}{\partial x_{q}}-\Gamma_{k}^{m q} A^{k} .
$$

Lowering the index $q$ with $g_{\ell q}$, we obtain the covariant 
derivative in the Hamilton manifold, with connection form (with the help of (16))

$$
\Gamma_{H \ell k}^{m} \equiv g_{\ell q} \Gamma_{k}^{m q}=\frac{1}{2} g^{m q}\left\{\frac{\partial g_{\ell q}}{\partial x^{k}}-\frac{\partial g_{k q}}{\partial x^{\ell}}-\frac{\partial g_{k \ell}}{\partial x^{q}}\right\} .
$$

This induced connection form, in the formula for curvature, would give a curvature corresponding to the Hamilton manifold. However, it is antisymmetric in its lower indices $(\ell, k)$ (torsion). Taken along a line parametrized by $t$, corresponding to geodesic motion, the antisymmetric terms cancel, leaving precisely the symmetric connection form (19). Note that (19) and (21) are not directly derived from $g_{i j}$; they are not metric compatible connections. However, performing parallel transport on the local flat tangent space of the Gutzwiller manifold, the resulting connection, after raising the tensor index to reach the Hamilton manifold, results in exactly the "truncated" connection (19). A complete discussion of the tensors on the Gutzwiller and the Hamilton manifolds will be given elsewhere. We note here, however, that the curvature associated with the geodesic deviation in the Hamilton manifold, as we shall see below, is not the same as the intrinsic curvature of that manifold, determined by $\Gamma_{H \ell k}^{m}$, but rather, due to the presence of torsion, a special curvature form associated with the geodesics themselves.

Since the coefficients $M_{m n}^{\ell}$ constitute a connection form, they can be used to construct a covariant derivative. It is this covariant derivative which must be used to compute the rate of transport of the geodesic deviation $\xi^{\ell}=x^{\prime \ell}-$ $x^{\ell}$ along the (approximately common) motion of neighboring orbits in the Hamilton manifold, since it follows the geometrical structure of the geodesics.

The second order geodesic deviation equations (substituting the conformal metric (7) into (22), and taking into account the constraint that both trajectories $x^{\ell \ell}$ and $x^{\ell}$ have the same energy $E$, one sees that (22) becomes the orbit deviation equation based on (14))

$$
\ddot{\xi}^{\ell}=-2 M_{m n}^{\ell} \dot{x}^{m} \dot{\xi}^{n}-\frac{\partial M_{m n}^{\ell}}{\partial x^{q}} \dot{x}^{m} \dot{x}^{n} \xi^{q},
$$

obtained from (18), can be factorized in terms of this covariant derivative,

$$
\xi_{; n}^{\ell}=\frac{\partial \xi^{\ell}}{\partial x^{n}}+M_{n m}^{\ell} \xi^{m} .
$$

One obtains

$$
\frac{D_{M}^{2}}{D_{M} t^{2}} \xi^{\ell}=R_{M q m n}^{\ell} \dot{x}^{q} \dot{x}^{n} \xi^{m},
$$

where the index $M$ refers to the connection (19), and what we shall call the dynamical curvature is given by

$$
R_{M q m n}^{\ell}=\frac{\partial M_{q m}^{\ell}}{\partial x^{n}}-\frac{\partial M_{q n}^{\ell}}{\partial x^{m}}+M_{q m}^{k} M_{n k}^{\ell}-M_{q n}^{k} M_{m k}^{\ell} .
$$

This expression, as remarked above, is not the curvature of the Hamilton manifold (given by this formula with $\Gamma_{H q m}^{\ell}$ in place of $M_{q m}^{\ell}$ ), but a dynamical curvature which is appropriate for geodesic motion.

We give in the following a general formula for the geodesic deviation in the Hamilton manifold in two dimensions (the resulting conditions can be easily generalized to three dimensions, for which they are also effective), and then show results of computer simulation for Poincaré plots showing a correspondence with the prediction of instability from the geodesic deviation.

With the conformal metric in noncovariant form (7) and (8), the dynamical curvature (25) can be written in terms of derivatives of the potential $V$, and the geodesic deviation Eq. (24) becomes

$$
\frac{D_{M}^{2} \xi}{D_{M} t^{2}}=-\mathcal{V P} \xi
$$

where the matrix $\mathcal{V}$ is given by

$$
\mathcal{V}_{\ell i}=\left\{\frac{3}{M^{2} v^{2}} \frac{\partial V}{\partial x^{\ell}} \frac{\partial V}{\partial x^{i}}+\frac{1}{M} \frac{\partial^{2} V}{\partial x^{\ell} \partial x^{i}}\right\} .
$$

and

$$
P^{i j}=\delta^{i j}-\frac{v^{i} v^{j}}{v^{2}},
$$

with $v^{i} \equiv \dot{x}^{i}$, defining a projection into a direction orthogonal to $v^{i}$.

We then find for the component orthogonal to the motion

$$
\frac{D_{M}^{2}\left(\mathbf{v}_{\perp} \cdot \xi\right)}{D_{M} t^{2}}=-\left[\lambda_{1} \cos ^{2} \phi+\lambda_{2} \sin ^{2} \phi\right]\left(\mathbf{v}_{\perp} \cdot \xi^{\ell}\right)
$$

where $\lambda_{1}$ and $\lambda_{2}$ are eigenvalues of the matrix $\mathcal{V}$, and $\phi$ is the angle between $\mathbf{v}_{\perp}$ and the eigenvector for $\lambda_{1}$.

Instability should occur if at least one of the eigenvalues of $\mathcal{V}$ is negative, in terms of the second covariant
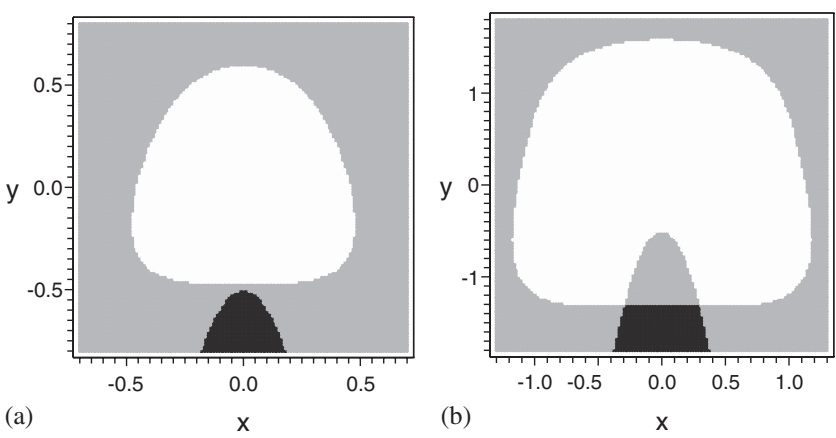

FIG. 1. (a) The dark area shows the region of negative eigenvalues for the matrix $\mathcal{V}$ for a Hamiltonian with potential (30). The light area corresponds to physically allowable motion for $E=1 / 6$. The region of negative eigenvalues does not penetrate the physically accessible region in this case. (b) The dark area of negative eigenvalues for the matrix $V$ is seen to penetrate deeply into the light region of physically allowable motion for $E=3$. 


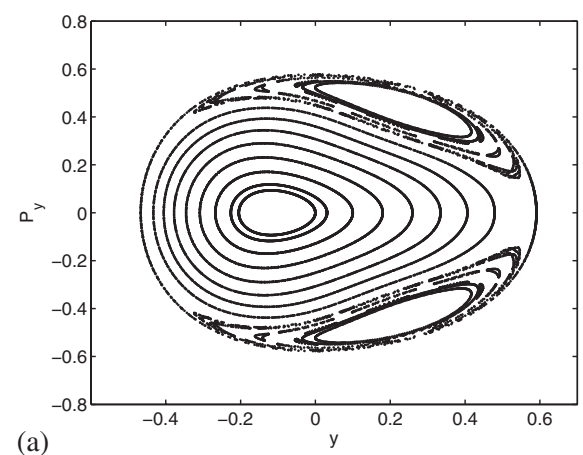

(a)

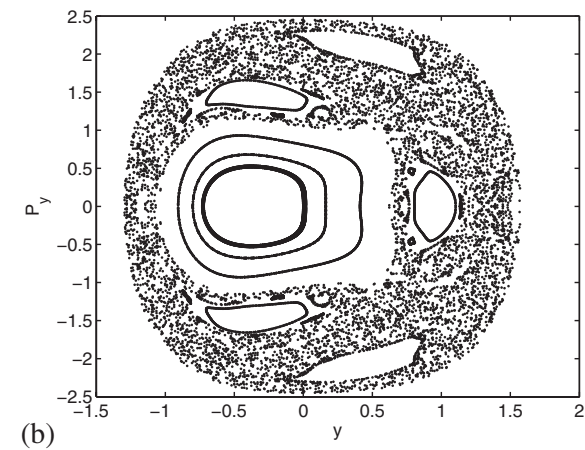

FIG. 2. (a) A Poincaré plot in the $(y$, $p_{y}$ ) plane for $E=1 / 6$, indicating regular motion. (b) A Poincaré plot in the $\left(y, p_{y}\right)$ plane for $E=3$, indicating a strongly chaotic behavior. derivatives of the transverse component of the geodesic deviation.

One may easily verify that the oscillator potential is predicted to be stable. Our criteria imply that the Duffing oscillator (without perturbation, not a chaotic system) clearly indicates instability in a neighborhood of the unstable fixed point. The potentials discussed by Oloumi and Teychenne [6] also demonstrate the effectiveness of our procedure; our results in these cases are in agreement with theirs. The relation (29) provides a clear indication of the local regions of instability giving rise to chaotic motion in the Hénon-Heiles model (this result will be discussed in detail elsewhere).

We take for a simple illustration here a slight modification of the fifth order expansion of a two body Toda lattice Hamiltonian (for which the fourth order expansion coincides with the Hénon-Heiles model)

$$
V(x, y)=\frac{1}{2}\left(x^{2}+y^{2}\right)+x^{2} y-\frac{1}{3} y^{3}+\frac{3}{2} x^{4}+\frac{1}{2} y^{4} .
$$

This provides a new Hamilton chaotic system for which our criterion gives a clear local signal for the presence of instability. Figure 1(a) shows that the region of negative eigenvalues does not penetrate the physically accessible region for $E=1 / 6$; Fig. 2(a) shows a Poincaré plot in the $y, p_{y}$ plane for this case, indicating completely regular orbits. In Fig. 1(b), the distribution of negative eigenvalues for $E=3$ is shown to penetrate deeply into the physical region, and Fig. 2(b) shows the corresponding Poincare plot displaying a high degree of chaotic behavior. The criterion for instability we have given depends sensitively on the energy of the system. The critical energy for which the negative eigenvalues begin to penetrate the physically accessible region, in this example, is $E \cong 1 / 5$, also the energy of transition for the Poincaré plot.

The condition implied by the geodesic deviation Eq. (26), in terms of covariant derivatives, in which the orbits are viewed geometrically as geodesic motion, is a new condition for instability, based on the underlying geometry, for a Hamiltonian system of the form (6). This geometrical picture of Hamiltonian dynamics provides, moreover, new insight into the structure of the unstable and chaotic behavior of Hamiltonian dynamical systems.

We wish to thank S. Shnider, A. Belenkiy, P. Leifer, I. Aharonovitch, and Avi Gershon for helpful discussions.

[1] C. G.J. Jacobi, Vorlesungen über Dynamik (Verlag Reimer, Berlin, 1884); J. S. Hadamard, J. Math. Pures Appl. 4, 27 (1898).

[2] M.C. Gutzwiller, Chaos in Classical and Quantum Mechanics (Springer-Verlag, New York, 1990). See also W. D. Curtis and F. R. Miller, Differentiable Manifolds and Theoretical Physics (Academic Press, New York, 1985); J. Moser and E. J. Zehnder, Notes on Dynamical Systems (Amer. Math. Soc., Providence, 2005); L. P. Eisenhardt, A Treatise on the Differential Geometry of Curves and Surfaces , (Ginn, Boston, 1909) (republished by Dover, N.Y., 2004)].

[3] V.I. Arnold, Mathematical Methods of Classical Mechanics (Springer-Verlag, New York, 1978).

[4] P. Appell, Dynamique des Systemes Mecanique Analytique (Gauthier-Villars, Paris, 1953); H. Cartan, Calcul Differential et Formes Differentielle (Herman, Paris, 1967); L. D. Landau, Mechanics (Mir, Moscow, 1969). The method was utilized for the relativistic case by D. Zerzion, L. P. Horwitz, and R. Arshansky, J. Math. Phys. (N.Y.) 32, 1788 (1991).

[5] L. Casetti and M. Pettini, Phys. Rev. E 48, 4320 (1993); L. Caiani, L. Casetti, C. Clementi, and M. Pettini, Phys. Rev. Lett. 79, 4361 (1997); L. Casetti, C. Clementi, and M. Pettini, Phys. Rev. E 54, 5969 (1996). See also, M. Szydlowski and J. Szczesny, Phys. Rev. D 50, 819 (1994); M. Szydlowski and A. Krawiec, Phys. Rev. D 53, 6893 (1996), who have studied a somewhat generalized Gutzwiller form (with the addition of a scalar potential) which accommodates the Jacobi metric.

[6] Atta Oloumi and Denis Teychenne, Phys. Rev. E 60, R6279 (1999). 